\title{
Titanium Carbide MXene Nucleation Layer for Epitaxial Growth of High-Quality GaN
}

\section{Nanowires on Amorphous Substrates}

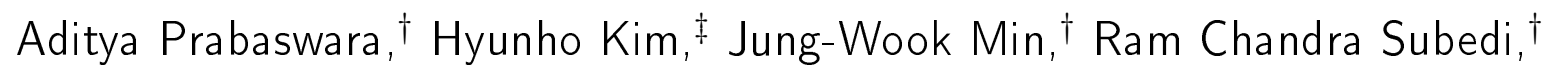

Dalaver H. Anjum, $\boldsymbol{\Phi}, \|$ Bambar Davaasuren, "Kalani Moore, ${ }^{\S}$ Michele Conroy, ${ }^{\S}$

Somak Mitra, ${ }^{\ddagger}$ Iman S. Roqan, ${ }^{\ddagger}$ Tien Khee Ng, ${ }^{* \dagger \dagger}$ Husam N. Alshareef, ${ }^{*} \ddagger$ and Boon S. Ooi*,†

$\dagger$ Computer, Electrical, and Mathematical Sciences and Engineering Division, King Abdullah

University of Science and Technology (KAUST), Thuwal 23955-6900, Saudi Arabia

$\ddagger$ Physical Science and Engineering Division, King Abdullah University of Science and Technology (KAUST), Thuwal 23955-6900, Saudi Arabia

ๆCore Labs, King Abdullah University of Science and Technology (KAUST), Thuwal

23955-6900, Saudi Arabia

$\S$ Department of Physics, Bernal Institute, University of Limerick, Limerick, V94 T9PX, Ireland

||Department of Physics, PO BOX 127788, Khalifa University, Abu Dhabi, United Arab Emirates

E-mail: tienkhee.ng@kaust.edu.sa; husam.alshareef@kaust.edu.sa; boon.ooi@kaust.edu.sa

\begin{abstract}
Growing III-nitride nanowires on 2D materials is advantageous as it effectively decouples the underlying growth substrate from the properties of the nanowires. As a
\end{abstract}


relatively new family of $2 \mathrm{D}$ materials, MXenes are promising candidates as III-nitride nanowire nucleation layers capable of providing simultaneous transparency and conductivity. In this work, we demonstrate the direct epitaxial growth of GaN nanowires on $\mathrm{Ti}_{3} \mathrm{C}_{2}$ MXene films. The MXene films consist of nanoflakes spray coated onto an amorphous silica substrate. We observed an epitaxial relationship between the GaN nanowires and the MXene nanoflakes due to the compatibility between the triangular lattice of $\mathrm{Ti}_{3} \mathrm{C}_{2}$ MXene and the hexagonal structure of wurtzite GaN. The GaN nanowires on MXene show good material quality and partial transparency at visible wavelengths. Nanoscale electrical characterization using conductive atomic force microscopy (C-AFM) reveals a Schottky barrier height of $\sim 330 \mathrm{meV}$ between the GaN nanowire and the $\mathrm{Ti}_{3} \mathrm{C}_{2}$ MXene film. Our work highlights the potential of using MXene as a transparent and conductive pre-orienting nucleation layer for high quality GaN growth on amorphous substrates.

\section{Keywords}

nanowires, MXene, molecular beam epitaxy, GaN, amorphous substrate

III-nitride nanowires have attracted much attention because of their potential as the main component in optoelectronic devices. Threading dislocation-free nanowires can be grown with reduced strain on various substrates without the requirement of a global epitaxial relationship. ${ }^{112}$ However, the fabrication of nanowire-based optoelectronic devices typically requires an electrically conductive substrate, which limits the substrate selection.

A possible method of circumventing the substrate limitation is using a $2 \mathrm{D}$ material as the nanowire nucleation layer. By utilizing a 2D material as a nucleation layer, the growth of the nanowires is effectively decoupled from the properties of the underlying substrate. The concept of growing III-nitride films and nanostructures on 2D materials including hexag-

onal boron nitride, ${ }^{3+5}$ graphene,,$\sqrt[6]{9}$ and transition metal dichalcogenides (TMDs) such as $\mathrm{MoS}_{2} \frac{10111}{10}$ has been demonstrated. The epitaxial relationship between III-nitride and the 2D 
material nucleation layer is generally assumed to be based on the van der Waals epitaxy mechanism, allowing high-quality material growth despite the lattice mismatch between the 2D material and III-nitride layers. ${ }^{12}$ By taking advantage of this growth property, it is possible to reproduce the growth process on any substrate capable of withstanding the growth process temperature.

Currently, graphene is arguably the most widely studied 2D material. However, the production of gaphene thin films is restricted by cost and size limitations in the direct chemical vapor deposition (CVD) synthesis process. Although another deposition method using spray-coating, spin-coating, and dip-coating of reduced graphene oxide (GO) has been

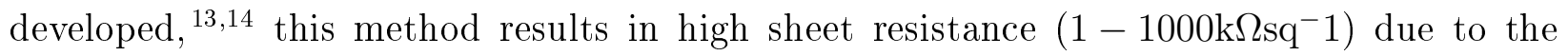
high inter-flake resistance and defects. ${ }^{15}$ Thus, the application of graphene for large-scale applications is limited by these factors.

As a new class of 2D materials, MXenes are thought to be viable alternatives for graphene. $\frac{16-20}{20}$ MXenes have several advantageous properties, such as high metallic conductivity and partial transparency. $\frac{21}{2}$ The potential for large scale transparent and conductive MXene films has been demonstrated through the spray coating of delaminated $\mathrm{Ti}_{3} \mathrm{C}_{2}$ nanoflakes, where the transparency can be adjusted from $40 \%$ to $90 \%$, while the sheet resistance ranges from 0.5 to $8 \mathrm{k} \Omega \mathrm{sq}^{-} 1.22 \mid 23$ This makes MXenes attractive as a transparent conductive layer for large scale applications. The triangular geometry of $\mathrm{Ti}_{3} \mathrm{C}_{2}$ MXene's basal plane $(\mathrm{a}=3.071 \AA)^{24}$ match well with that of GaN (3.189 $\AA$ ), which helps promote the growth of high-quality GaN on top of $\mathrm{Ti}_{3} \mathrm{C}_{2}$ MXene. The van der Waals epitaxial growth mode of materials such as $\mathrm{BaTiO}_{3}$ on top of MXene has also been demonstrated, 25 which may support applications such as the transfer of GaN nanowires on MXene onto foreign substrates. $\frac{26}{}$ The work function of heat-treated $\mathrm{Ti}_{3} \mathrm{C}_{2}$ MXene $(4.28 \mathrm{eV})^{27}$ is comparable to the work function of $\mathrm{Ti}(4.33 \mathrm{eV})$, which is a metal commonly used to form ohmic contact to $n$-GaN. Currently, no III-nitride growth on MXene has been reported. Thus, the study of III-nitride nanowire growth on MXene is an interesting topic, as it will introduce another possible 2D material family for 
the growth and fabrication of III-nitride devices.

In this work, we demonstrate the epitaxial growth of III-nitride nanowires directly on $\mathrm{Ti}_{3} \mathrm{C}_{2}$ MXene using plasma-assisted molecular beam epitaxy (PA-MBE). We observed an epitaxial relationship between the MXene film and the in-plane orientation of the GaN nanowires, which suggests that the triangular lattice of the $\mathrm{Ti}_{3} \mathrm{C}_{2}$ MXene plays a role in adjusting the in-plane orientation of the GaN nanowires. Our analysis shows that MXenes are promising as transparent and conductive nucleation layers for epitaxial growth of GaN nanowires on amorphous substrates.

\section{Results and Discussion}

The structure of GaN nanowires grown directly on $\mathrm{Ti}_{3} \mathrm{C}_{2}$ MXene nanoflakes is depicted in Figure 1 (a). To evaluate the feasibility of using MXene film for high-temperature growth we loaded a fused silica substrate coated with $\mathrm{Ti}_{3} \mathrm{C}_{2}$ MXene film into the MBE buffer chamber and annealed it. The annealing was performed at $750{ }^{\circ} \mathrm{C}$ under a base pressure of $1 \times 10^{-9}$ Torr, which is a typical growth temperature of GaN by MBE. After the annealing process was complete, we performed a Raman spectroscopy on the MXene film. All Raman measurements were performed under a non-polarized backscattering configuration using a Horiba Aramis system with a $473 \mathrm{~nm}$ laser as an excitation source. The effect of annealing is shown

in Figure 1 (b). From the Raman spectra, we see that MXene peak softening occurs. This softening might be attributed to the relocation of oxygen surface functional groups into sites that were previously occupied by the fluorine surface groups before ultra-high vacuum annealing. The desorption of fluorine has been observed after annealing at this temperature.28 These changes in the Ti-O bond configuration can alter the Raman active vibration modes, resulting in peak softening..$^{29}$

We further investigated the effect of $\mathrm{Ti}_{3} \mathrm{C}_{2}$ MXene film on the morphology and quality of the GaN nanowires. We grew GaN nanowires directly on top of bare fused silica , on 
a partially covered MXene film, and on a fully covered MXene film as the nucleation layer under nominally identical conditions. The root mean square (rms) roughness for the fused silica, partially covered MXene film, and fully covered MXene film are $0.79 \mathrm{~nm}, 3.5 \mathrm{~nm}$, and $5 \mathrm{~nm}$ respectively. By interpolating the transmittance measurement data with known thickness vs transmittance values, ${ }^{22}$ we determine the fully covered MXene film to be about $19.2 \mathrm{~nm}$ thick. The growth was performed at $800^{\circ} \mathrm{C}$, with a $2.5 \times 10^{-8}$ Torr gallium beam equivalent pressure, and radio frequency (RF) plasma at $350 \mathrm{~W}$ power and 1 sccm nitrogen flow rate. The Raman spectra are shown in Figure 1 (c), while the plan view scanning electron microscopy (SEM) images are shown in Figure 1 (d)-(f).

We investigated the $E_{\text {high }}^{2}$ phonon mode associated with the wurtzite GaN structure $30 \mid 31$ by Raman spectroscopy to evaluate the quality of the GaN nanowires. GaN nanowires grown directly on fused silica grow in a highly dense manner with a high degree of coalescence as shown in Figure 1 (d). The nanowires grow with a random in-plane orientation, which is typical for nanowire growth on amorphous silica surface. $\frac{32133}{3}$ The relative intensity of the $E_{\text {high }}^{2}$ phonon is weaker than the signal from the fused silica, due to the poor quality of the GaN material.

Figure 1 (e) shows the plan view of GaN nanowires grown on a partially covered MXene film. In general, the GaN nanowires coalesce into a compact layer, while in some areas, the nanowires grow with a morphology similar to the morphology of nanowires grown directly on fused silica. Atomic force microscopy (AFM) measurements on the surface (see inset) shows that the fused silica is only partially covered with MXene in a nonuniform manner, resulting in nanowires growing directly on the exposed fused silica. On the other hand, the nanowires grow with high density on top of the MXene nanoflakes, eventually coalescing into a compact layer with hexagonal features on the surface. Figure 1 (f) shows the plan view of GaN nanowires grown on a fully covered MXene film. A clear nanowire morphology with hexagonal facets can be seen. The diameter and density of the nanowires grown on MXene are non-uniform across the substrate surface. Our data show a decrease in nanowire 
density with an increasing number of MXene flakes (controlled by changing the number of MXene spray coated layers), which is similar that observed for GaN nanowires grown on multilayer graphene. ${ }^{34}$ As the number of MXene flakes is increased, the nucleation probability of nanowires is decreased, which is caused by elasticity and strain partitioning between the GaN nucleus and the underlying layered material. Thus, the thickness of the MXene film can be used to control the morphology of the GaN nanowires grown on top. Although the fully covered MXene film shows some thickness fluctuations, the fluctuations are considered to be too small to create a significant nanowire morphology variation.
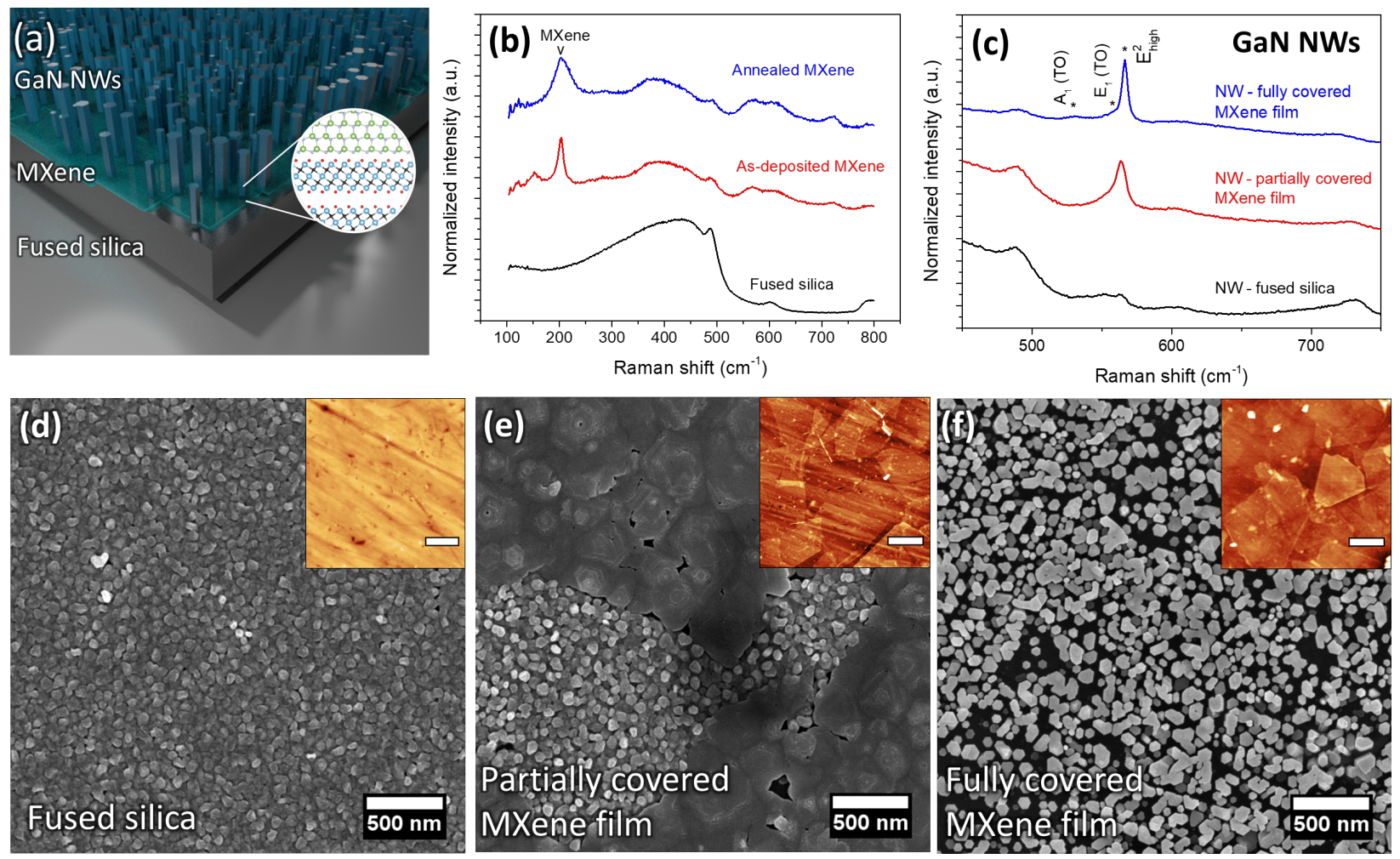

Figure 1: (a) Depiction of the GaN nanowires (NWs) grown on MXene film. (b) Raman spectra comparison among bare fused silica, an as-deposited MXene film, and a MXene film after annealing inside the MBE buffer chamber. (c) Raman spectra comparison among GaN NWs grown on top of bare fused silica, partially covered MXene film, and fully covered MXene film. Plan-view SEM image of nanowires grown on (d) bare fused silica; (e) partially covered MXene film; and (f) fully covered MXene film. The inset shows the AFM micrograph of the corresponding substrate surface, with a scale bar corresponding to $1 \mu \mathrm{m}$.

Elevated and cross-section view of the GaN nanowires grown on the fully covered MXene film are shown in Figure 2 (a) and (b), respectively. The nanowires grow perpendicular to the 
substrate, with some tilting caused by the uneven surface of the MXene film. The alignment of the nanowires and the interplanar spacing between the MXene monolayers were evaluated using polycrystalline X-ray diffraction (XRD) as shown in Figure 2 (c). The $\theta / 2 \theta$ scan of the GaN nanowire sample shows a dominant GaN(0002) peak and additional GaN(0004) peak, which indicates growth in the c-plane direction typical of spontaneously grown GaN nanowires. The inset shows the $\mathrm{Ti}_{3} \mathrm{C}_{2}$ (0002) peak, which can be ascribed to the interlayer spacing of the MXene nanoflakes. After nanowire growth occurs, the c lattice parameter of the MXene film decreases as shown by the shift in the $\mathrm{Ti}_{3} \mathrm{C}_{2}$ (0002) peak. This might be caused by the removal of the intercalated water molecules between the MXene layers during high-temperature annealing in the ultra high vacuum environment. The verticality of the GaN nanowire ensemble is evaluated using the XRD rocking curve of the GaN(0002) peak, shown in Figure 2 (d). The (0002) peak has full width at half maximum (FWHM) of $5925 \operatorname{arcsec}\left(1.646^{\circ}\right)$, which is comparable to the FWHM value for GaN nanowires grown on multilayer graphene. $\frac{34}{2}$ The broad FWHM value comes from the misorientation of the GaN nanowire's verticality due to the thickness variation of the MXene film and variations among the nanowire heights.

From the plan view SEM in Figure 3(a), we observe that nanowires grown close together in a small area exhibit similar in-plane orientations. A lower magnification SEM in Figure 3 (b) shows multiple in-plane orientations, as indicated by the false-color map on the SEM image. This is caused by the epitaxial relationship between the nanowires and the underlying MXene nanoflakes. GaN nanowires that grow on the same MXene nanoflake show identical in-plane orientation, which indicates an epitaxial relationship between the first atomic plane of the GaN nanowires and the $\mathrm{Ti}_{3} \mathrm{C}_{2}$ MXene.

We performed an XRD measurement on a $1 \times 1 \mathrm{~cm}^{2}$ sample to obtain the pole figure of the GaN $\{10-14\}$ reflection. While GaN nanowires grown on $\mathrm{Si}(111)$ typically exhibit sixfold symmetry, $\stackrel{35}{25 i g u r e ~ 3}$ (c) shows that the GaN nanowires grown on MXene nanoflakes do not exhibit this behavior. Instead, an almost random pattern with some preferred in-plane 

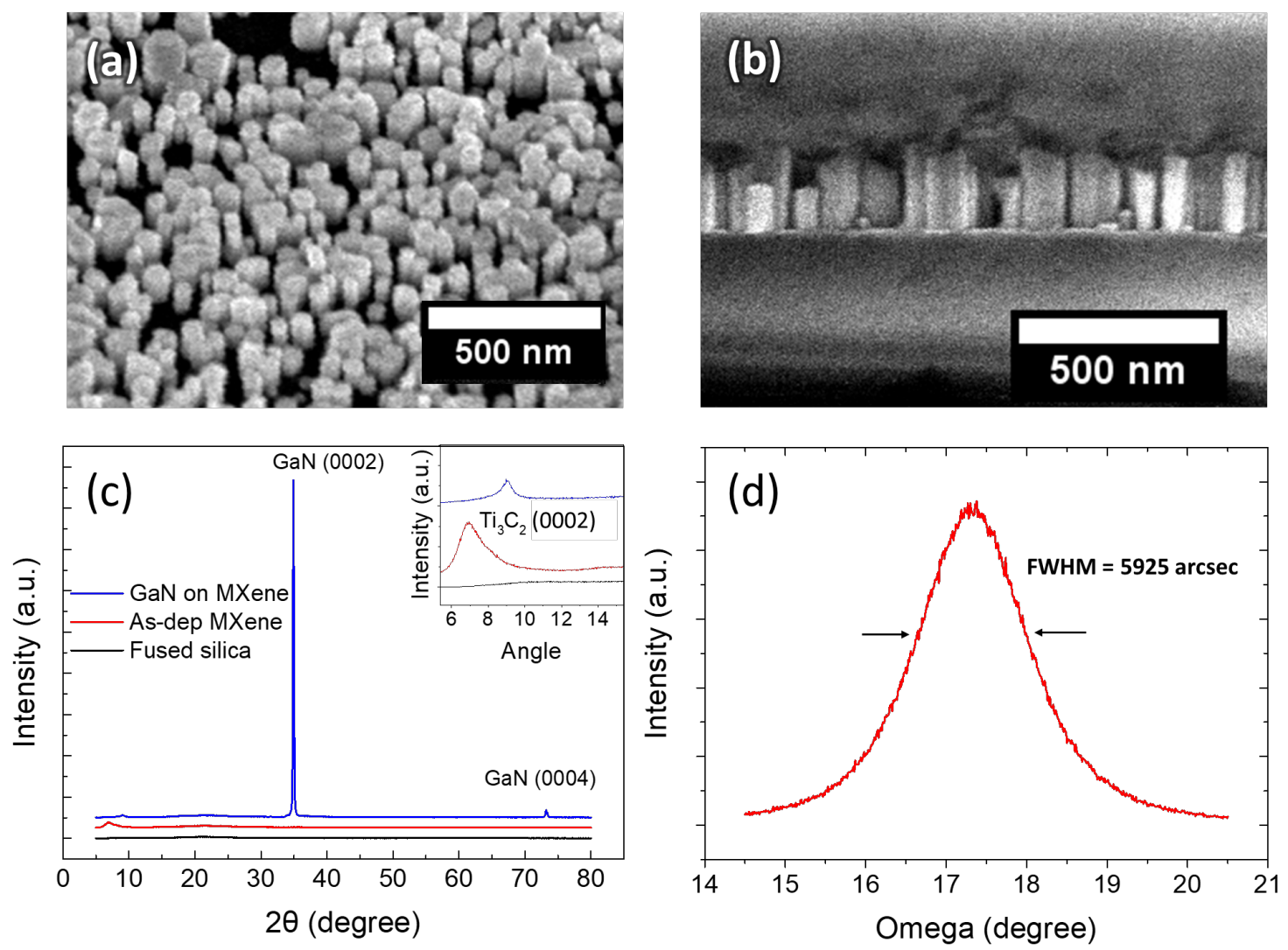

Figure 2: (a) Elevated and (b) cross-section SEM micrograph of the GaN nanowires grown on fully covered MXene film. (c) The $\theta / 2 \theta$ XRD curves of the fused silica, as-deposited MXene, and GaN nanowires on MXene. The inset shows a higher resolution graph around the $\mathrm{Ti}_{3} \mathrm{C}_{2}$ (0002) peak. (b) XRD Rocking curve of the GaN (0002) peak. 
orientation can be seen. The lack of symmetry is attributed to the random in-plane orientation distribution of MXene nanoflakes, which were spray coated on top of the amorphous substrate.
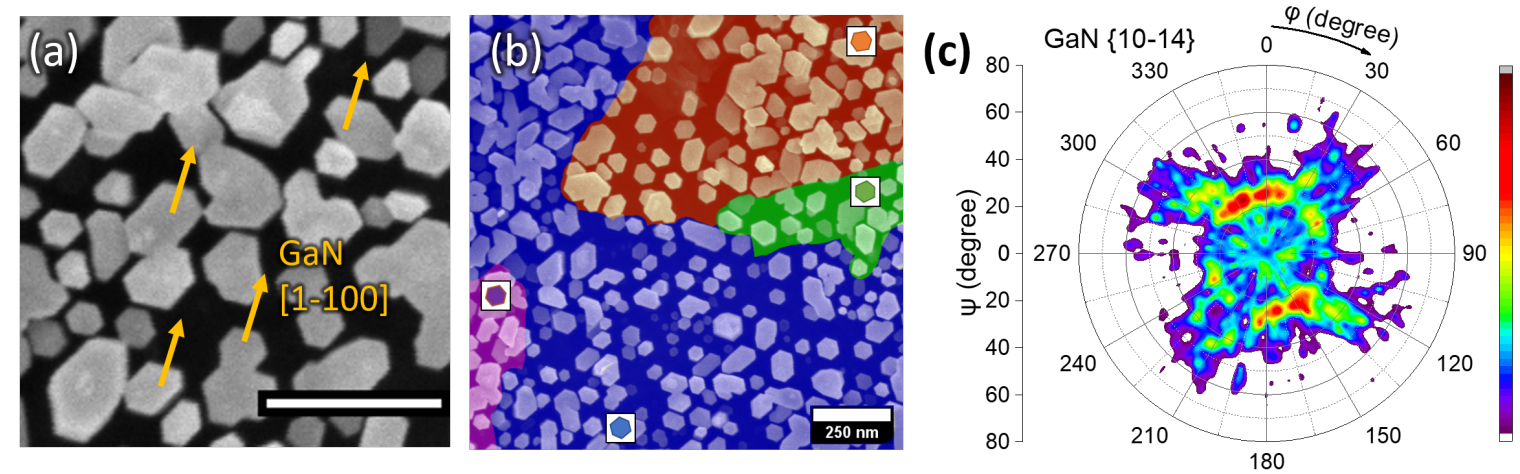

Figure 3: Effect of MXene nanoflakes on the in-plane orientation of GaN nanowires. (a) Plan-view of GaN nanowires grown on MXene. The nanowires within the small area exhibit identical in-plane orientation. The scale bar corresponds to $200 \mathrm{~nm}$. (b) Lower magnification plan-view SEM showing distinct in-plane orientations of the nanowires, as indicated by the false color map. (c) XRD pole figure plot taken from the $\{10-14\}$ reflection of GaN, showing an almost random in-plane orientation distribution.

The structural properties of GaN nanowires and the interface between the GaN and MXene film were studied using transmission electron microscopy (TEM). Figure 4 (a) shows a low-magnification TEM of the GaN nanowires. A selective area diffraction pattern image was taken of the GaN nanowires, showing single crystallinity. A higher-magnification TEM image showing a single GaN nanowire grown directly on top of the MXene film is shown in Figure 4(b). The MXene film consists of individual MXene nanoflakes layered on top of each other in a random manner, resulting in a non-uniform film thickness across the substrate. This can be seen by the presence of a step at the base of the nanowire.

The horizontal lines indicated in Figure 4(b) correspond to an area with a high density of Type $I_{1}$ basal stacking faults (BSFs). This stacking fault is typically modeled as the insertion of a thin zincblende phase within the wurtzite GaN nanowire matrix. BSFs have been observed in MBE-grown III-ntiride nanowires grown on $\mathrm{Si}\left[\frac{36}{36}\right.$ and $\mathrm{Ti}$ foil,,$\sqrt[37]{3}$ and are

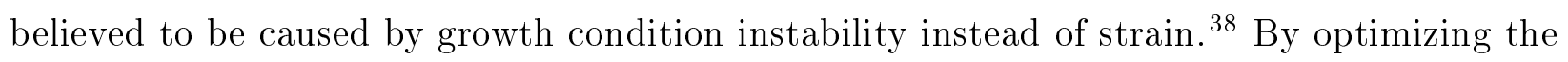


growth condition, it is possible to prevent the formation of BSFs.

A high-resolution high-angle annular dark field (HAADF) image of the interface between the nanowires and MXene is shown in Figure 4 (c). The GaN nanowires grow in the $<0001>$ c-plane direction perpendicular to the substrate and MXene film. The GaN nanowire grows directly on the MXene film without the formation of a polycrystalline wetting layer which has been observed for GaN nanowires grown on nitridized silicon substrate and fused silica substrate. $\frac{32139}{\text { Figure } 4}$ (d) shows an HAADF image of the GaN-MXene film-fused silica interface with the energy-dispersive X-ray (EDX) elemental mapping result superimposed. We captured the EDX signal from $\mathrm{Ga}$, Ti, and $\mathrm{Si}$, which corresponds to the GaN nanowires, MXene film, and fused silica layers, respectively. A clear interface can be seen among the $\mathrm{Ga}, \mathrm{Ti}$, and Si signals.

Based on the known lattice constant of both wurtzite $\mathrm{GaN}$ and $\mathrm{Ti}_{3} \mathrm{C}_{2}$ MXene, we simulated the possible heteroepitaxial relationship between the wurtzite GaN nanowires and the underlying MXene film. As shown in Figure 4 (e,f), the distance between the nearest GaN primitive unit cell along the $\langle 11-20\rangle$ direction is $3.189 \AA$, while the distance between $\mathrm{Ti}$ atoms along the $\langle 11-20\rangle$ direction is $3.071 \AA$. This configuration gives a lattice mismatch of $\sim 3.7 \%$, allowing for coherent strain between the GaN nanowires and the $\mathrm{Ti}_{3} \mathrm{C}_{2}$ MXene film.

Based on the structural characterization that we have performed, $\mathrm{Ti}_{3} \mathrm{C}_{2}$ MXene is suitable for the growth of GaN nanowires due to the compatibility between the triangular atomic geometry of MXene and the wurtzite structure of GaN. Due to the surface functional group on the surface of the $\mathrm{Ti}_{3} \mathrm{C}_{2}$ MXene nanoflakes, the MXene film has a negative polarity. This negative polarity may serve a similar function as the N-polar surface of AlN nucleation layers for promoting the initial nucleation for nanowire growth. ${ }^{40}$ The surface functional groups consist of oxygen, hydroxyl, and halogen groups; the latter subject to desorption during annealing in ultrahigh vacuum environment. The remaining oxygen and hydroxyl groups can rearrange their site, creating local fluctuation of surface chemistry that can act as nucle- 

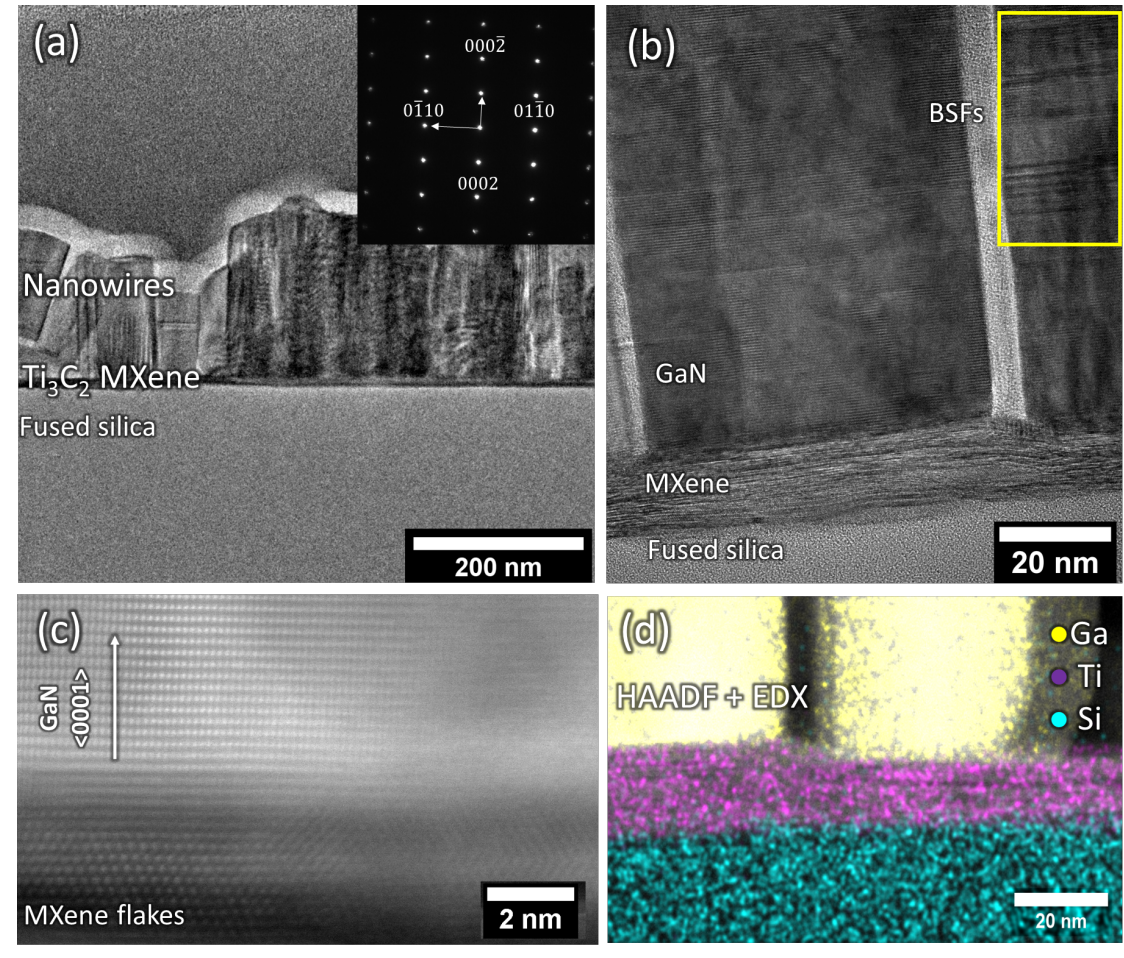

(e)

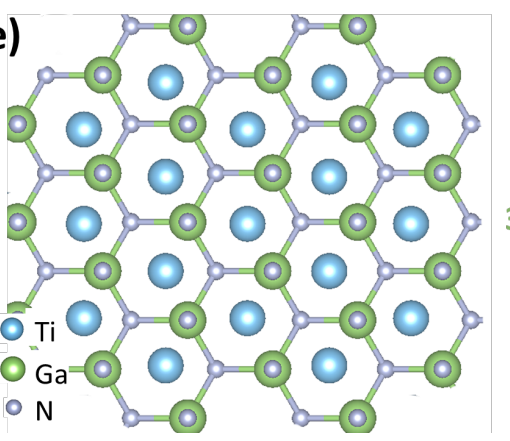

(f)

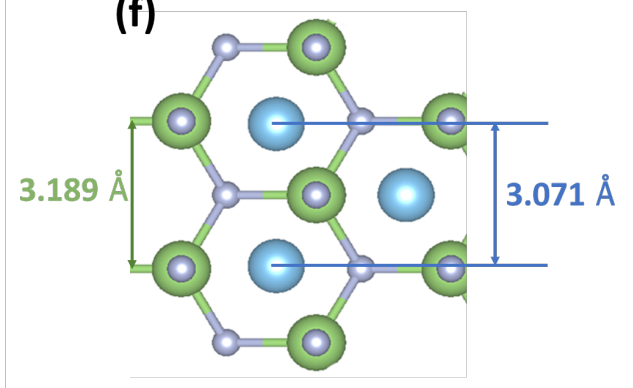

Figure 4: (a) Low magnification TEM images of GaN nanowires grown on $\mathrm{Ti}_{3} \mathrm{C}_{2} \mathrm{MXene}$. The inset shows the selective area diffraction pattern taken at the GaN nanowires. (b) A high-resolution TEM image GaN nanowire, showing the direct growth of the GaN nanowires on top of the MXene film. The presence of BSFs is indicated by the yellow box. (c) Highresolution HAADF image taken at the interface between the MXene film and GaN base, showing GaN growth in the $<0001>$ direction. (d) A composite HAADF and EDX elemental mapping image showing the composition of Ga, Ti, and Si. (e,f) Schematic images of the atomic configuration representing the heteroepitaxial relationship between the first atomic plane of wurtzite $\mathrm{GaN}$ and $\mathrm{Ti}_{3} \mathrm{C}_{2}$ Mxene. 
ation sites. Thus, $\mathrm{Ti}_{3} \mathrm{C}_{2}$ may be more advantageous than graphene in promoting nanowire nucleation. However, considering how GaN nanowires can be grown on both polar (N-polar $\mathrm{AlN}$ ) and non-polar (graphene) ${ }^{[34}$ nucleation layer using PA-MBE, the surface polarity might not be the major factor in determining the nucleation of GaN nanowires. Nonetheless, the possible effect of the polarity of MXene and surface functional groups on the nucleation and growth kinetics of GaN nanowires cannot be ruled out.

Transparent devices can be beneficial for applications requiring simultaneous transparency and conductivity, such as photovoltaic solar cells and photoelectrochemical water splitting. The transmittance value for the MXene film and GaN nanowires grown on MXene was evaluated using UV-Vis spectrophotometry, as shown in Figure 5(a), with corresponding optical photographs of the samples shown in (b) and (c). For the MXene film, the transmittance value is above $40 \%$ for the entire visible wavelength region. After the growth of the GaN nanowires occured, the transmittance value was reduced. One of the possible causes is light scattering from the ensemble nanowire structure. ${ }^{41}$ Hantanasirisakul et al. also observed the relationship between light transmittance through the $\mathrm{Ti}_{3} \mathrm{C}_{2}$ MXene film and the c-lattice spacing between the MXene nanoflakes, where a larger lattice spacing generally leads to a higher transmittance. Therefore, the reduced MXene c-plane spacing after GaN nanowire growth might also contribute to the reduced transmittance value. ${ }^{22}$ This also highlights the possibility of tuning the transmittance value of the device after growth by introducing an intercalant between the MXene nanoflakes.

To evaluate the optical properties along with the material quality of the GaN nanowires grown on MXene, we performed photoluminescence (PL) measurements at room temperature (RT) and low temperature $(4 \mathrm{~K})$ and $244 \mathrm{~nm}$ laser excitation. ${ }^{42}$ The PL results are summarized in Figure 5 (d) and (e). The RT PL emission peak is broad with an FWHM of $\sim 40 \mathrm{meV}$, with multiple peaks visible at low temperature $(4 \mathrm{~K})$. Peak deconvolution using the Gaussian peak fitting routine reveals 4 major peaks (FWHM) centered at $3.46 \mathrm{eV}$ (7.34 $\mathrm{meV}), 3.47 \mathrm{eV}(10.44 \mathrm{meV}), 3.49 \mathrm{eV}(19.42 \mathrm{meV})$, and 3.51 (8.11 mev) respectively. Slightly 

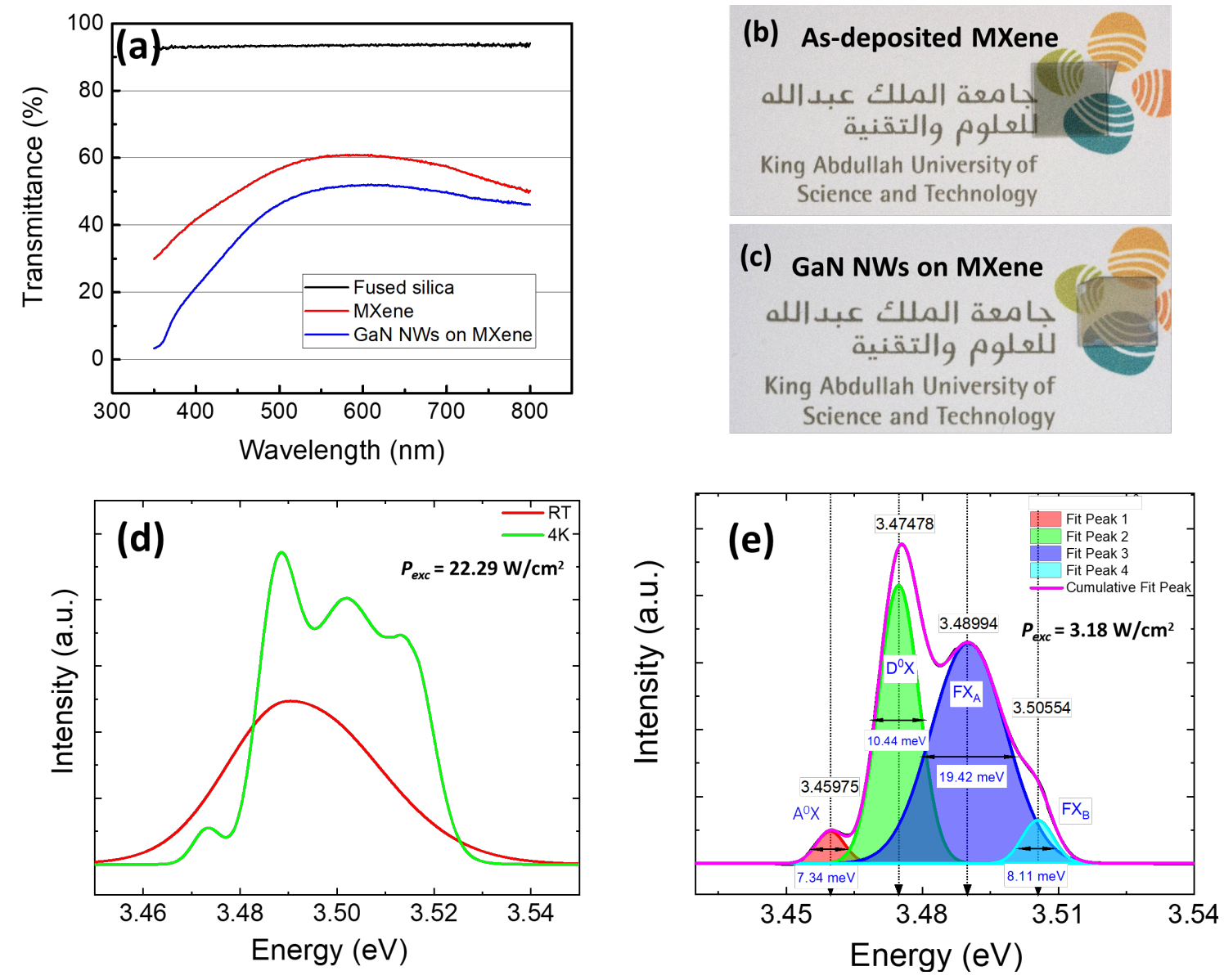

Figure 5: (a) UV-Vis transmittance measurement for the fused silica, MXene film, and GaN nanowires on MXene. (b,c) corresponding optical photograph of the samples. (d) RT and 4 K PL spectra of the nanowires on MXene sample. (e) Peak deconvolution of the PL spectra at $4 \mathrm{~K}$ using a Gaussian peak fitting routine. 
altered peak positions and FWHM values can be caused by scattered binding energy values of the excitons resulting from the different growth conditions, the crystallinity of the substrates, residual strain in the heteroepitaxial GaN layers, residual unintentional dopants in the growth chambers, and the excitation power density used. $\frac{32|34| 43-46}{4}$ Low-temperature PL is expected to be dominated by neutral donor-bound exciton transitions $\left(\mathrm{D}^{0} \mathrm{X}\right)$, which we observe at $3.47 \mathrm{eV}$ whereas the peak at the lower energy side of $\mathrm{D}^{0} \mathrm{X}$ centered at $3.46 \mathrm{eV}$ with an FWHM of $7.34 \mathrm{meV}$ is attributed to the acceptor-bound excitons $\left(\mathrm{A}^{0} \mathrm{X}\right) .{ }^{46}$ Even though the GaN nanowires are $n$-doped, the existence of acceptor-bound excitons can be attributed to the residual impurities in the MBE chamber. $\frac{34|45| 46}{3}$ The two peaks on the higher energy side of $\mathrm{D}^{0} \mathrm{X}$ are recognized as free exciton related emission, namely $\mathrm{FX}_{\mathrm{A}}$ and $\mathrm{FX}_{\mathrm{B}}$. The FWHM of the dominant PL peak, $\mathrm{D}^{0} \mathrm{X}$, in our case is $10.44 \mathrm{meV}$, which is narrower than

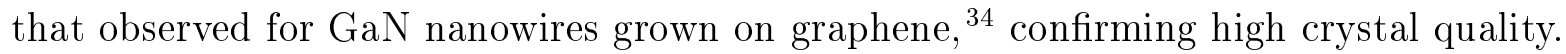

Typically, the fabrication of nanowire-based devices on insulating substrates such as silica requires additional processing steps such as the use of flip-chip configuration. ${ }^{47}$ By using MXene as the nucleation layer for the nanowires, lateral conduction across the insulating substrate is supported. For electrical characterization, the nanowires are probed using conductive AFM (C-AFM). The top of the $n$-doped nanowires is contacted using a PtIr coated conductive AFM tip without prior. Apart from the standard solvent cleaning procedure, no chemical treatments were performed on the GaN nanowires. In this configuration, the C-AFM tip is grounded, while a bias is applied to the MXene film. The MXene film is electrically contacted to the AFM stage by attaching a copper wire to the area where GaN nanowires do not grow. A simplified schematic of the C-AFM setup is shown in Figure 6 (a). In this setup, a negative current value indicates current flowing from the C-AFM tip to the substrate. We performed a voltage sweep from -6 to $6 \mathrm{~V}$, and the tip current was limited from -10 to $10 \mathrm{nA}$. Therefore, structural damage due to junction heating in the nanowire is unlikely.

The PtIr C-AFM tip, the $n$-GaN nanowire, and the MXene film arrangement create a 
metal-semiconductor-metal (MSM) configuration. In the case of heavily doped $n$-type GaN nanowires, thermionic field emission is the dominant mechanism for carrier transport through the Schottky barrier. ${ }^{48}$

Figure 6 (b) shows the I-V curve obtained from random sampling of multiple nanowires. In general, the turn-on voltage under reverse bias (RB) is lower than the turn-on voltage under forward bias (FB). This can be explained by the difference in the Schottky barrier height at each end of the nanowire, as depicted in Figure 6 (c). To extract the Schottky barrier height at both ends of the nanowires, we performed an MSM fitting routine using the PKUMSM MATLAB script. In the model, the C-AFM measurement configuration is considered to be two Schottky barriers connected back to back, with the nanowire as a resistor in series with the Schottky barriers. ${ }^{49}$ To simplify the fitting process, we used an average nanowire diameter of $60 \mathrm{~nm}$ and a height of $150 \mathrm{~nm}$. The effect of applied voltage on the Schottky barrier height is taken into account; thus the fitting routine gives an effective Schottky barrier height. The fitting result is shown in Figure 6 (c). From the fitting parameters, the Schottky barrier height between $n$-GaN and MXene is approximately $330 \mathrm{meV}$, while the barrier height between $n$-GaN and the PtIr tip is approximately $840 \mathrm{meV}$.

The large Schottky barrier height between the C-AFM tip and $n$-GaN is expected, as the work function of the PtIr C-AFM tip $(\sim 5.1 \mathrm{eV})$ is significantly larger than the electron affinity $\chi$ of GaN $(4.1 \mathrm{eV}) .{ }^{50151}$ However, the observed value of the Schottky barrier is lower than $\phi_{P t I r}-\chi_{G a N}(\sim 1 \mathrm{eV})$. This difference is caused by the effect of the nanometer-sized contact between the GaN nanowire and the PtIr tip. As the tip of the C-AFM probe (25 $\mathrm{nm})$ is smaller than the average diameter of the nanowires $(60 \mathrm{~nm})$, the Schottky metal contact can become unstable, depending on the applied tip force. Furthermore, Jamond et al. demonstrated that the Schottky contact area formed between the C-AFM tip and the nanowire is approximately a few nanometers in size. As the contact area is much smaller than the depletion width in GaN nanowires, the conventional Schottky diode description cannot be used .50 Furthermore, the GaN nanowires did not undergo chemical treatment before the 
C-AFM measurement, which may result in a modified surface potential due to the presence of native a surface oxide layer.

On the other hand, the interface between the GaN nanowire and $\mathrm{Ti}_{3} \mathrm{C}_{2}$ MXene shows a Schottky contact behavior despite $\mathrm{Ti}_{3} \mathrm{C}_{2}$ Mxene and Ti having a similar work function. During the nanowire growth process inside the MBE chamber, we cannot rule out the possibility of carbon or titanium interdiffusion into the GaN nanowire, which may form an interfacial layer or result in unintentional doping at the base of the nanowires. This formation of the interfacial layer may explain the Schottky contact at the interface of the GaN nanowires and the MXene. Exposure to nitrogen plasma has also been shown to induce doping effects in $\mathrm{MoS}_{2},{ }^{[52}$ which may alter the work function of the MXene film. The Schottky barrier behavior may help in fabricating Schottky-based devices such as piezotronic energy harvesting devices. By adjusting the doping concentration of the $n$-GaN nanowire, it is also possible to promote electron tunneling through the Schottky barrier for optoelectronic device applications.
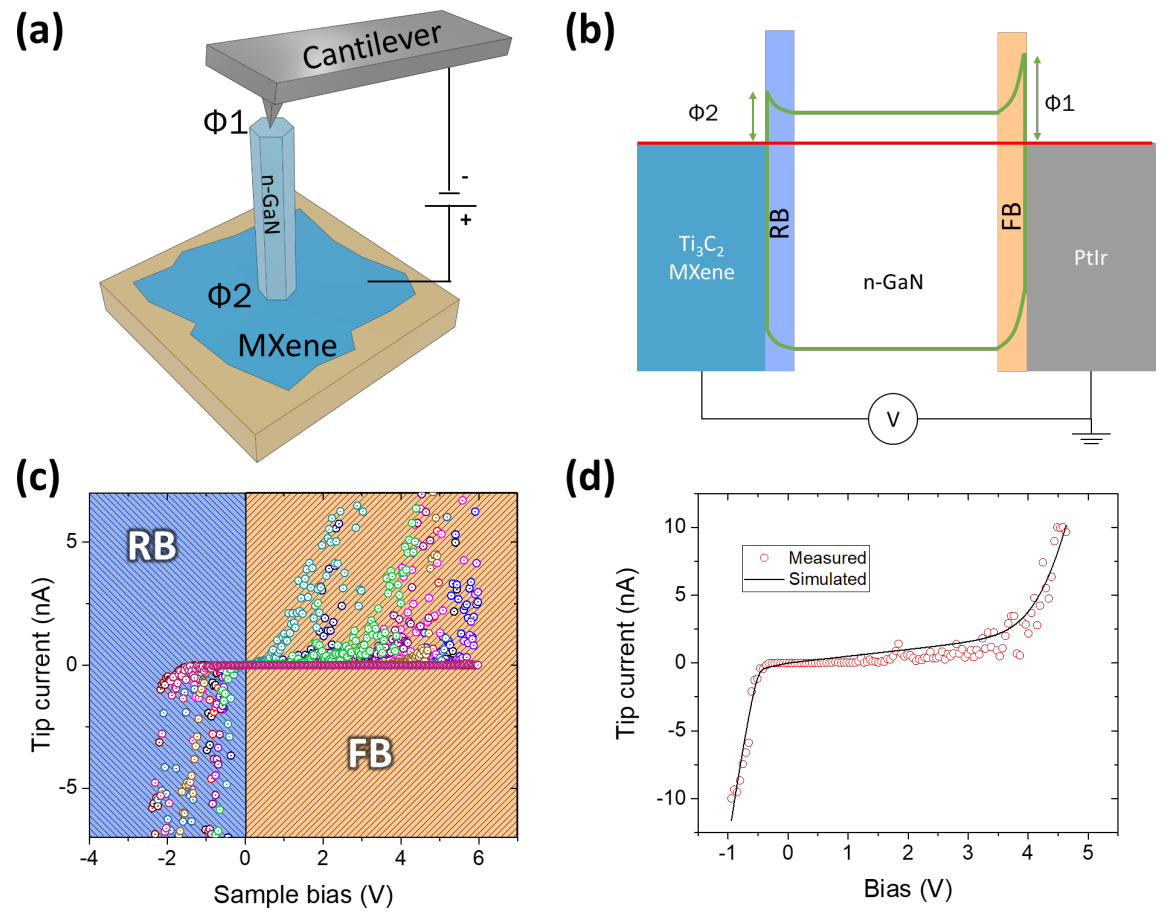

(d)

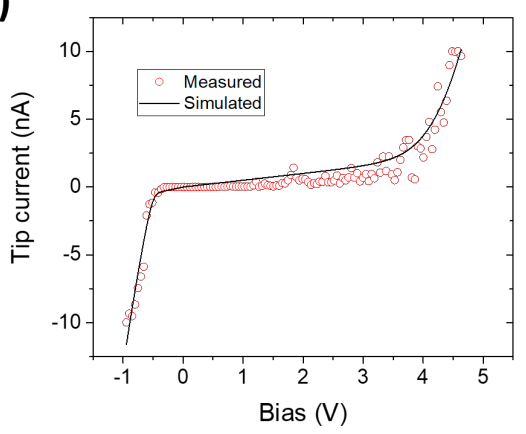

Figure 6: (a) Schematic for the C-AFM measurement setup. (b) Simplified band diagram schematic of the metal-semiconductor-metal interface formed by the C-AFM tip, $n$-GaN nanowire, and MXene film under equilibrium. (c) I-V curves obtained from multiple individual nanowires. (d) I-V Curve fitting using PKUMSM routine. 


\section{Conclusions}

We have shown that $\mathrm{Ti}_{3} \mathrm{C}_{2}$ MXene is suitable as a nucleation layer for GaN nanowire growth on amorphous substrates due to the low lattice mismatch between MXene and GaN nanowires $(\sim 3.7 \%)$. The MXene nanoflakes can be deposited on a large surface area through the spray coating method, making it attractive for large scale applications. By adjusting the thickness of the MXene film and the growth conditions of the GaN nanowires, a high-quality nanowire structure can be grown directly on MXene films without the need for buffer layers. The epitaxial relationship between $\mathrm{Ti}_{3} \mathrm{C}_{2}$ MXene and GaN resulted in an ordered in-plane orientation of the GaN nanowires regardless of the orientation of the underlying substrate. Nanoscale electrical measurements using C-AFM show a Schottky barrier of approximately $330 \mathrm{meV}$ between the GaN nanowire and $\mathrm{Ti}_{3} \mathrm{C}_{2}$ MXene film. The transparency, electrical conductivity, and scalability of the GaN nanowire/MXene platform make it attractive for applications such as transparent electronics, solar cells, energy harvesting, and photoelectrochemical water splitting.

\section{Methods}

$\mathrm{Ti}_{3} \mathrm{C}_{2}$ MXene synthesis: $\mathrm{Ti}_{3} \mathrm{AlC}_{2}$ (layered ternary carbide MAX phase) powder was purchased from Carbon-Ukraine Ltd. (particle size $<40 \mu \mathrm{m}$ ). One gram of $\mathrm{Ti}_{3} \mathrm{AlC}_{2} \mathrm{MAX}$ phase powder was slowly immersed into a mixture solution of $20 \mathrm{ml} 9 \mathrm{M} \mathrm{HCl}$ and $1 \mathrm{~g}$ of LiF under magnet stirring that was cooled by an ice bath to minimize localized heat from the initial exothermic reaction. The mixture solution was kept at $35^{\circ} \mathrm{C}$ with magnet stirring in an oil bath for 24 hours. The mixture was transferred to a centrifuge tube and washed several times with additional deionized (DI) water to make a total volume of $50 \mathrm{ml}$. Each wash was performed by centrifugation at 3000 relative centrifugal force (RCF) for 5 min. The supernatant was decanted, and the sediment was redispersed into DI water by manual shaking. Once the $\mathrm{pH}$ of the supernatant reached approximately 6 , the final centrifugation 
step was performed at $500 \mathrm{RCF}$ for 30 min after redispersing the sediment in DI water. The supernatant solution containing delaminated 2D MXene nanoflakes was collected and was used for spray coating.

Spray coating of MXene: Double side polish 2" fused silica (Semiconductor Wafer, Inc.) was diced into $1 \times 1 \mathrm{~cm}^{2}$ size followed by ultrasonic cleaning by acetone, isopropanol, and dried by nitrogen flow. The fused silica substrate is then treated by UV/ozone treatment for 10 minutes to enhance the surface hydrophilicity. Aqueous $\mathrm{Ti}_{3} \mathrm{C}_{2}$ MXene suspension with their concentration of $\sim 1.0-1.5 \mathrm{mg} / \mathrm{ml}$ was sprayed by airbrush (HB-B plus, Iwata, Japan) with a fluid nozzle of $0.2 \mathrm{~mm}$, assisted by pressurized nitrogen gas at $\sim 3.5-4.0$ bar ( $\sim 50-60 \mathrm{psi})$. The spray gun was positioned at $\sim 15-20 \mathrm{~cm}$ away from the substrates. The spray coating was performed in a layer-by-layer manner under mild-warm airflow to accelerate drying until the desired thickness is reached.

III-Nitride nanowire growth: MXene-coated fused silica was cleaned using acetone and isopropyl alcohol, followed by blow drying under nitrogen. No water was used during the cleaning procedure to prevent MXene film delamination. A Veeco Gen 930 MBE chamber with thermal effusion cells and RF plasma source was used. Thermal cleaning was performed inside the MBE preparation chamber at $650^{\circ} \mathrm{C}$ under a background pressure of $1 \times 10^{-9}$ Torr. GaN nanowire growth is performed inside the MBE growth chamber under nitrogen-rich conditions. The nucleation and growth of $n$-GaN was performed using a single growth step at $800^{\circ} \mathrm{C}$. Throughout the growth process, the nitrogen plasma condition was maintained at 1 sccm flow rate with 350 watt $\mathrm{RF}$ power.

Photoluminescence measurement: PL measurements were carried out using a $244 \mathrm{~nm}$ continuous wave laser, and the emission signal was detected by an Andor Shamrock 303i spectrograph attached to an Andor Newton CCD detector assisted with 299 lines/mm grating blazed at $300 \mathrm{~nm}$, where the laser spot size was focused to $\sim 100 \mu \mathrm{m}$. The acquisition time used was 1 second for a 10 measurement integration. A closed cycle refrigerator ARS cryostat was employed for cryogenic measurements. PL spectra comparison between RT and $4 \mathrm{~K}$ was 
performed with a PL excitation power density of $22.29 \mathrm{~W} \mathrm{~cm}^{-2}$, and $4 \mathrm{~K}$ spectra acquisition for deconvolution was performed under a PL excitation power density of $3.18 \mathrm{~W} \mathrm{~cm}^{-2}$.

Raman spectroscopy: Raman spectroscopy was performed in an unpolarized backscattering configuration using a Horiba Aramis system with a $473 \mathrm{~nm}$ laser excitation.

\section{Acknowledgement}

We acknowledge the financial support from the King Abdulaziz City for Science and Technology (KACST) under grant no. KACST TIC R2-FP-008. This work was partially supported by the King Abdullah University of Science and Technology (KAUST) baseline funding, and MBE equipment funding no. C/M-20000-12-001-77 and KCR/1/4055-01-01. The authors thank L.-M. Peng of Peking State University for providing the PKUMSM MATLAB script.

\section{References}

1. Colby, R.; Liang, Z.; Wildeson, I. H.; Ewoldt, D. A.; Sands, T. D.; Garcia, R. E.; Stach, E. A. Dislocation Filtering in GaN Nanostructures. Nano Lett. 2010, 10, 15681573.

2. Zhao, C.; Alfaraj, N.; Chandra Subedi, R.; Liang, J. W.; Alatawi, A. A.; Alhamoud, A. A.; Ebaid, M.; Alias, M. S.; Ng, T. K.; Ooi, B. S. III-Nitride Nanowires on Unconventional Substrates: From Materials to Optoelectronic Device Applications. Prog. Quantum Electron. 2018, 61, 1-31.

3. Kobayashi, Y.; Kumakura, K.; Akasaka, T.; Makimoto, T. Layered Boron Nitride as a Release Layer for Mechanical Transfer of GaN-Based Devices. Nature 2012, 484, 223227.

4. Ayari, T.; Sundaram, S.; Li, X.; El Gmili, Y.; Voss, P. L.; Salvestrini, J. P.; Ougazzaden, A. Wafer-Scale Controlled Exfoliation of Metal Organic Vapor Phase Epitaxy 
Grown InGaN/GaN Multi Quantum Well Structures Using Low-Tack Two-Dimensional Layered h-BN. Appl. Phys. Lett. 2016, 108, 171106.

5. Sundaram, S.; Li, X.; Halfaya, Y.; Ayari, T.; Patriarche, G.; Bishop, C.; Alam, S.; Gautier, S.; Voss, P. L.; Salvestrini, J. P.; Ougazzaden, A. Large-Area van der Waals Epitaxial Growth of Vertical III-Nitride Nanodevice Structures on Layered Boron Nitride. Adv. Mater. Interfaces 2019, 190020\%, 1900207.

6. Chung, K.; Beak, H.; Tchoe, Y.; Oh, H.; Yoo, H.; Kim, M.; Yi, G.-C. Growth and Characterizations of GaN Micro-Rods on Graphene Films for Flexible Light Emitting Diodes. APL Mater. 2014, 2, 092512.

7. Kim, J.; Bayram, C.; Park, H.; Cheng, C.-W.; Dimitrakopoulos, C.; Ott, J. a.; Reuter, K. B.; Bedell, S. W.; Sadana, D. K. Principle of Direct van der Waals Epitaxy of Single-Crystalline Films on Epitaxial Graphene. Nat. Commun. 2014, 5, 4836.

8. Heilmann, M.; Munshi, A. M.; Sarau, G.; Göbelt, M.; Tessarek, C.; Fauske, V. T.; van Helvoort, A. T. J.; Yang, J.; Latzel, M.; Hoffmann, B.; Conibeer, G.; Weman, H.; Christiansen, S. Vertically Oriented Growth of GaN Nanorods on Si Using Graphene as an Atomically Thin Buffer Layer. Nano Lett. 2016, 16, 3524-3532.

9. Høiaas, I. M.; Liudi Mulyo, A.; Vullum, P. E.; Kim, D.-C.; Ahtapodov, L.; Fimland, B.o.; Kishino, K.; Weman, H. GaN/AlGaN Nanocolumn Ultraviolet Light-Emitting Diode Using Double-Layer Graphene as Substrate and Transparent Electrode. Nano Lett. 2019, 19, 1649-1658.

10. Zhao, C.; Ng, T. K.; Tseng, C.-C.; Li, J.; Shi, Y.; Wei, N.; Zhang, D.; Consiglio, G. B.; Prabaswara, A.; Alhamoud, A. A.; Albadri, A.; Alyamani, A. Y.; Zhang, X. X.; Li, L.-J.; Ooi, B. S. InGaN/GaN Nanowires Epitaxy on Large-Area MoS2 for High-Performance Light-Emitters. $R S C A d v$. 2017, 7, 26665-26672. 
11. Tangi, M.; Mishra, P.; Li, M. Y.; Shakfa, M. K.; Anjum, D. H.; Hedhili, M. N.; Ng, T. K.; Li, L. J.; Ooi, B. S. Type-I Band Alignment at MoS2/In0.15A10.85N Lattice Matched Heterojunction and Realization of MoS2 Quantum Well. Appl. Phys. Lett. 2017, 111, $0-5$.

12. Yu, J.; Wang, L.; Hao, Z.; Luo, Y.; Sun, C.; Wang, J.; Han, Y.; Xiong, B.; Li, H. Van der Waals Epitaxy of III-Nitride Semiconductors Based on 2D Materials for Flexible Applications. Adv. Mater. 2019, 1903407, 1903407.

13. Song, Y. I.; Yang, C.-M.; Kim, D. Y.; Kanoh, H.; Kaneko, K. Flexible Transparent Conducting Single-Wall Carbon Nanotube Film With Network Bridging Method. J. Colloid Interface Sci. 2008, 318, 365-371.

14. Tung, V. C.; Allen, M. J.; Yang, Y.; Kaner, R. B. High-Throughput Solution Processing of Large-Scale Graphene. Nat. Nanotechnol. 2009, 4, 25-29.

15. De, S.; Coleman, J. N. Are There Fundamental Limitations on the Sheet Resistance and Transmittance of Thin Graphene Films? ACS Nano 2010, 4, 2713-2720.

16. Hantanasirisakul, K.; Gogotsi, Y. Electronic and Optical Properties of 2D Transition Metal Carbides and Nitrides (MXenes). Adv. Mater. 2018, 30, 1-30.

17. Kim, H.; Wang, Z.; Alshareef, H. N. MXetronics: Electronic and Photonic Applications of MXenes. Nano Energy 2019, 60, 179-197.

18. Anasori, B.; Lukatskaya, M. R.; Gogotsi, Y. 2D Metal Carbides and Nitrides (MXenes) for Energy Storage. Nat. Rev. Mater. 2017, 2, 16098.

19. Barsoum, M. W.; Hultman, L.; Carle, J.; Lu, J.; Mashtalir, O.; Presser, V.; Naguib, M.; Gogotsi, Y. Two-Dimensional Transition Metal Carbides. ACS Nano 2012, 6, 1322-1331.

20. Naguib, M.; Mochalin, V. N.; Barsoum, M. W.; Gogotsi, Y. 25th Anniversary Article: MXenes: A New Family of Two-Dimensional Materials. Adv. Mater. 2014, 26, 992-1005. 
21. Zhang, C. J.; Nicolosi, V. Graphene and MXene-Based Transparent Conductive Electrodes and Supercapacitors. Energy Storage Mater. 2019, 16, 102-125.

22. Hantanasirisakul, K.; Zhao, M.-Q.; Urbankowski, P.; Halim, J.; Anasori, B.; Kota, S.; Ren, C. E.; Barsoum, M. W.; Gogotsi, Y. Fabrication of Ti3C2Tx MXene Transparent Thin Films with Tunable Optoelectronic Properties. Adv. Electron. Mater. 2016, 2, 1600050.

23. Wang, Z.; Kim, H.; Alshareef, H. N. Oxide Thin-Film Electronics using All-MXene Electrical Contacts. Adv. Mater. 2018, 1706656, 1-7.

24. Kurtoglu, M.; Naguib, M.; Gogotsi, Y.; Barsoum, M. W. First Principles Study of TwoDimensional Early Transition Metal Carbides. MRS Commun. 2012, 2, 133-137.

25. Bennett-Jackson, A. L.; Falmbigl, M.; Hantanasirisakul, K.; Gu, Z.; Imbrenda, D.; Plokhikh, A. V.; Will-Cole, A.; Hatter, C.; Wu, L.; Anasori, B.; Gogotsi, Y.; Spanier, J. E. Van der Waals Epitaxy of Highly (111)-Oriented BaTiO3 on MXene. Nanoscale 2019, 11, 622-630.

26. Li, H.; Wu, J.; Huang, X.; Yin, Z.; Liu, J.; Zhang, H. A Universal, Rapid Method for Clean Transfer of Nanostructures onto Various Substrates. ACS Nano 2014, 8, 65636570 .

27. Fu, H. C.; Ramalingam, V.; Kim, H.; Lin, C. H.; Fang, X.; Alshareef, H. N.; He, J. H. MXene-Contacted Silicon Solar Cells with 11.5\% Efficiency. Adv. Energy Mater. 2019, 9, $1-9$.

28. El-Demellawi, J. K.; Lopatin, S.; Yin, J.; Mohammed, O. F.; Alshareef, H. N. Tunable Multipolar Surface Plasmons in 2D Ti3C2Tx MXene Flakes. ACS Nano 2018, 12, 84858493. 
29. Persson, I.; Näslund, L.-Å.; Halim, J.; Barsoum, M. W.; Darakchieva, V.; Palisaitis, J.; Rosen, J.; Persson, P. O. A. On the Organization and Thermal Behavior of Functional Groups on Ti3C2 Mxene Surfaces in Vacuum. 2D Mater. 2017, 5, 015002.

30. Davydov, V. Y.; Kitaev, Y. E.; Goncharuk, I.; Smirnov, A.; Graul, J.; Semchinova, O.; Uffmann, D. Phonon Dispersion and Raman Scattering in Hexagonal GaN and AlN. Phys. Rev. B: Condens. Matter Mater. Phys. 1998, 58, 12899-12907.

31. Harima, H. Properties of GaN and Related Compounds Studied by Means of Raman Scattering. J. Phys.: Condens. Matter 2002, 14, R967-R993.

32. Kumaresan, V.; Largeau, L.; Oehler, F.; Zhang, H.; Mauguin, O.; Glas, F.; Gogneau, N.; Tchernycheva, M.; Harmand, J.-C. Self-Induced Growth of Vertical GaN Nanowires on Silica. Nanotechnology 2016, 27, 135602.

33. Junaid, M.; Chen, Y. T.; Palisaitis, J.; Garbrecht, M.; Hsiao, C. L.; Persson, P. O.; Hultman, L.; Birch, J. Liquid-Target Reactive Magnetron Sputter Epitaxy of High Quality GaN(0001) Nanorods on Si(111). Mater. Sci. Semicond. Process. 2015, 39, 702-710.

34. Kumaresan, V.; Largeau, L.; Madouri, A.; Glas, F.; Zhang, H.; Oehler, F.; Cavanna, A.; Babichev, A.; Travers, L.; Gogneau, N.; Tchernycheva, M.; Harmand, J.-C. Epitaxy of GaN Nanowires on Graphene. Nano Lett. 2016, 16, 4895-4902.

35. Schuster, F.; Weiszer, S.; Hetzl, M.; Winnerl, A.; Garrido, J. A.; Stutzmann, M. Influence of Substrate Material, Orientation, and Surface Termination on GaN Nanowire Growth. J. Appl. Phys. 2014, 116 .

36. Goodman, K. D.; Protasenko, V. V.; Verma, J.; Kosel, T. H.; Xing, H. G.; Jena, D. Green Luminescence of InGaN Nanowires Grown on Silicon Substrates by Molecular Beam Epitaxy. J. Appl. Phys. 2011, 109, 084336. 
37. Calabrese, G.; Corfdir, P.; Gao, G.; Pfüller, C.; Trampert, A.; Brandt, O.; Geelhaar, L.; Fernández-Garrido, S. Molecular Beam Epitaxy of Single Crystalline GaN Nanowires on a Flexible Ti Foil. Appl. Phys. Lett. 2016, 108, 202101.

38. Zhou, X.; Lu, M.-Y.; Lu, Y.-J.; Jones, E. J.; Gwo, S.; Gradečak, S. Nanoscale Optical Properties of Indium Gallium Nitride/Gallium Nitride Nanodisk-in-Rod Heterostructures. ACS Nano 2015, 9, 2868-2875.

39. Stoica, T.; Sutter, E.; Meijers, R. J.; Debnath, R. K.; Calarco, R.; Lüth, H.; Grützmacher, D. Interface and Wetting Layer Effect on the Catalyst-Free Nucleation and Growth of GaN Nanowires. Small 2008, 4, 751-754.

40. Fernández-Garrido, S.; Kong, X.; Gotschke, T.; Calarco, R.; Geelhaar, L.; Trampert, A.; Brandt, O. Spontaneous Nucleation and Growth of GaN Nanowires: The Fundamental Role of Crystal Polarity. Nano Lett. 2012, 12, 6119-6125.

41. Diedenhofen, S. L.; Janssen, O. T. A.; Grzela, G.; Bakkers, E. P. A. M.; Gómez Rivas, J. Strong Geometrical Dependence of the Absorption of Light in Arrays of Semiconductor Nanowires. ACS Nano 2011, 5, 2316-2323.

42. Ajia, I. A.; Yamashita, Y.; Lorenz, K.; Muhammed, M. M.; Spasevski, L.; Almalawi, D.; Xu, J.; Iizuka, K.; Morishima, Y.; Anjum, D. H.; Wei, N.; Martin, R. W.; Kuramata, A.; Roqan, I. S. GaN/AlGaN Multiple Quantum Wells Grown on Transparent and Conductive (-201)-oriented $\beta$-Ga2O3 Substrate for UV Vertical Light Emitting Devices. Applied Physics Letters 2018, $113,082102$.

43. Reynolds, D. C.; Look, D. C.; Jogai, B.; Saxler, A. W.; Park, S. S.; Hahn, J. Y. Identification of the $\Gamma 5$ and $\Gamma 6$ Free Excitons in GaN. Appl. Phys. Lett. 2000, 77, 2879-2881.

44. Monemar, B.; Paskov, P. P.; Bergman, J. P.; Toropov, A. A.; Shubina, T. V.; Malinauskas, T.; Usui, A. Recombination of Free and Bound Excitons in GaN. Phys. Status Solidi B 2008, 245, 1723-1740. 
45. Xu, S. J.; Liu, W.; Li, M. F. Direct Determination of Free Exciton Binding Energy from Phonon-Assisted Luminescence Spectra in GaN Epilayers. Appl. Phys. Lett. 2002, 81, 2959-2961.

46. Na, S. H.; Kyhm, K. Time-Resolved Free Exciton Trapping and Thermal Activation in Bound-Exciton in GaN. J. Korean Phys. Soc. 2007, 51, 539-544.

47. Park, Y.; Jahangir, S.; Park, Y.; Bhattacharya, P.; Heo, J. InGaN/GaN Nanowires Grown on $\mathrm{SiO} 2$ and Light Emitting Diodes with Low Turn On Voltages. Opt. Express 2015, 23, A650.

48. Tangi, M.; Min, J. W.; Priante, D.; Subedi, R. C.; Anjum, D. H.; Prabaswara, A.; Alfaraj, N.; Liang, J. W.; Shakfa, M. K.; Ng, T. K.; Ooi, B. S. Observation of Piezotronic and Piezo-Phototronic Effects in n-InGaN Nanowires/Ti Grown by Molecular Beam Epitaxy. Nano Energy 2018, 54, 264-271.

49. Liu, Y.; Zhang, Z. Y.; Hu, Y. F.; Jin, C. H.; Peng, L.-M. Quantitative Fitting of Nonlinear Current-Voltage Curves and Parameter Retrieval of Semiconducting Nanowire, Nanotube and Nanoribbon Devices. J. Nanosci. Nanotechnol. 2008, 8, 252-258.

50. Jamond, N.; Chrétien, P.; Gatilova, L.; Galopin, E.; Travers, L.; Harmand, J. C.; Glas, F.; Houzé, F.; Gogneau, N. Energy Harvesting Efficiency in GaN Nanowire-Based Nanogenerators: The Critical Influence of the Schottky Nanocontact. Nanoscale 2017, 9, 4610-4619.

51. Schäffl, F. Properties of Advanced Semiconductor Materials : GaN, AlN, InN, BN, SiC, SiGe. John Wiley Sons Inc New York 2001,

52. Mishra, P.; Tangi, M.; Ng, T. K.; Hedhili, M. N.; Anjum, D. H.; Alias, M. S.; Tseng, C.C.; Li, L.-J.; Ooi, B. S. Impact of N-Plasma and Ga-Irradiation on MoS2 Layer in Molecular Beam Epitaxy. Appl. Phys. Lett. 2017, 110, 012101. 


\section{Graphical TOC Entry}

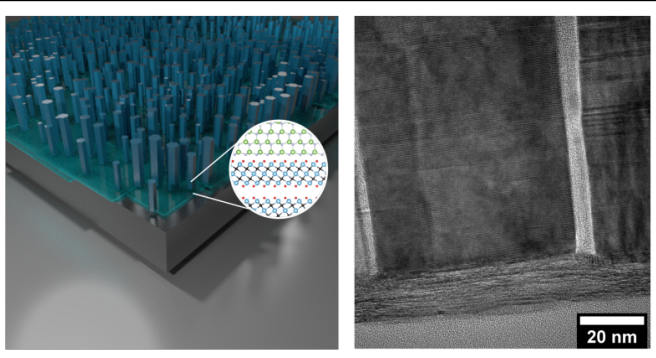

3D illustration and cross-section TEM image of GaN nanowire growing on MXene nanoflakes. 